\title{
PROLONGATION OF CONNECTIONS TO BUNDLES OF INFINITELY NEAR POINTS
}

\author{
AKIHIKO MORIMOTO
}

\section{Introduction}

The purpose of this paper is to generalize the results of [5] to the bundles of infinitely near points of $A$-kinds in the sense of A. Weil [7], which generalizes the notions of $p^{r}$-jets in the sense of C. Ehresmann [1], [2]. Our results naturally generalizes the results of several authors, e.g., [4], [8], [10]. In fact, we have treated the same problem in the author's lecture notes (cf. [6, Part V]). However, in [6] we fully used the basis and structure constants of the local algebra $A$, and were obliged to consider ( $\lambda$ )-lifts of vector fields, 1-forms or tensor fields of type $(p, q)$ with $p=0$ or 1 , where $\lambda=0,1,2, \cdots, N$ and $N+1=\operatorname{dim} A$. Moreover, the geometric meaning of $(\lambda)$-lifts for $\lambda=1,2$, $\cdots, N$ are not so clear as that of (0)-lifts. In this paper, we shall essentially not use the basis and structure constants of the algebra $A$, and shall show that there exists essentially only one lift, which has a significant geometric meaning, and other ( $\lambda$ )-lifts can be derived naturally from that lift. Further, the proofs in [6] are much simplified, and some of results are somewhat sharpened (cf. [6, Theorem 6.6]).

In $\S 1$, we explain the notion of local algebras and the infinitely near points of $A$-kind which will be simply called $A$-points. The covariant functor, which assigns to each manifold $M$ its bundle $M^{A}$ of infinitely near points, has many nice properties similar to the functor which assigns to $M$ its tangent bundle $T(M)$. In particular, if $G$ is a Lie group (acting on a manifold $M$ ), then $G^{A}$ is also a Lie group (acting on $M^{A}$ ).

In $\S 2$, by means of two different methods we define two $A$-module structures on the tangent space to $M^{A}$ at each point of $M^{A}$, and we shall in fact show that these two $A$-module structures are essentially the same.

In $\S 3$, we shall define the lift of vector fields and establish some relations between the lift of functions and the bracket of vector fields.

In $\S 4, \S 5$, we shall consider the lifting of covariant tensor fields and $(1,1)-$ tensor fields respectively. We shall prove that the lifting $J^{A}$ of an almost complex structure $J$ is integrable if and only if $J$ is integrable.

In $\S 6$, we shall first construct the prolongation of affine connections (Theorem 6.1), and next show that the prolonged affine connection $\nabla^{A}$ is locally

Received May 29, 1970, and, in revised form, December 6, 1975. 
affine symmetric if and only if $\nabla$ is.

In $\S 7$, we shall give a proof for the fact that if $M$ is an affine symmetric space then $M^{A}$ is also so. In such a manner, we obtain a method to construct a large number of affine symmetric spaces (resp. complex manifolds) from a given affine symmetric space (resp. complex manifold), (cf. [10, Introduction]).

In this paper, all manifolds and mappings (functions) are assumed to be differentiable of class $C^{\infty}$, unless otherwise stated.

The author wishes to thank Professor J. L. Koszul who gave him an opportunity to stay at the University of Grenoble and suggested the idea of $A$ module structure in $\S 2$, which has essentially improved [6, Part V].

\section{Infinitely near points of $A$-kind}

In this section we shall recall the notion of local algebras and infinitely near points of $A$-kind in the sense of A. Weil [7].

Definition 1.1. Let $A$ be an associative algebra over the field $R$ of real numbers with a unit (denoted by 1 ). We call $A$ a local algebra if $A$ is commutative and of finite dimension over $R$, admitting a unique maximal ideal $\mathfrak{m}$ such that $A / \mathfrak{m}$ is of dimension 1 over $R$ and that $\mathfrak{m}^{h+1}=(0)$ for a nonnegative integer $h$. The smallest $h$ such that $\mathfrak{m}^{h+1}=(0)$ will be called the height of $A$. We shall identify the field $\boldsymbol{R}$ with the subspace of $A$ consisting of all scalar multiples of the unit 1. Clearly $A$ is the direct sum of $\boldsymbol{R}$ and $\mathfrak{m}$ as a vector space. If $a \in A$, the scalar $a_{0} \in R$, defined by $a \equiv a_{0} \bmod \mathfrak{m}$, will be called the finite part of $a$. If $A / \mathrm{m}$ is identified with $R$, the map $a \rightarrow a_{0}$ is a homomorphism of $A$ onto $R$.

Let $R[p]=R\left[\left[X_{1}, \cdots, X_{p}\right]\right]$ be the algebra of all formal power series of $p$ indeterminates $X_{1}, \cdots, X_{p}$, and let $\mathfrak{m}_{p}$ be the maximal ideal of $R[p]$ consisting of all formal power series without constant terms. Let $\mathfrak{a}$ be an ideal of $R[p]$ such that $\operatorname{dim} R[p] / \mathfrak{a}<+\infty$. We see that $A=\boldsymbol{R}[p] / \mathfrak{a}$ is a local algebra with the maximal ideal $\mathfrak{m}=\mathfrak{m}_{p} / \mathfrak{a}$. Conversely, we know that every local algebra is isomorphic to such a local algebra (cf. [7, p. 112]).

Let $M$ be a manifold of dimension $n$, and let $C^{\infty}(M)$ be the algebra of all differentiable functions on $M$. Take a point $x \in M$.

Definition 1.2. Let $A$ be a local algebra with the maximal ideal $\mathrm{m}$. An algebra homomorphism $x^{\prime}: C^{\infty}(M) \rightarrow A$ will be called an $A$-point of $M$ near to $x$ (or infinitely near point to $x$ on $M$ of $A$-kind) if the finite part of $x^{\prime}(f)$ is equal to $f(x)$, i.e.,

$$
x^{\prime}(f) \equiv f(x) \quad \bmod \mathfrak{m}
$$

for every $f \in C^{\infty}(M)$. We denote by $M_{x}^{A}$ the set of all $A$-points of $M$ near to $x$ and $M^{A}=\bigcup_{x \in M} M_{x}^{A}$, and define $\pi_{A}: M^{A} \rightarrow M$ by $\pi_{A}\left(M_{x}^{A}\right)=x$ for $x \in M$.

Remark 1.3. If $x^{\prime} \in M_{x}^{A}$, and $f \in C^{\infty}(M)$ vanishes identically on a neighborhood of $x$, then we see that $x^{\prime}(f)=0$. 
This remark shows that we can consider $x^{\prime}(f)$ for any differentiable function $f$ defined on a neighborhood of $x$ if $x^{\prime} \in M_{x}^{A}$.

Remark 1.4. If we take $\mathfrak{a}=\left(\mathfrak{m}_{p}\right)^{r-1}$, and $A=R[p] / \mathfrak{a}$, then we see that the notion of $A$-points is nothing but the notion of $p^{r}$-jets (cf. [1], [2], [7]). In particular, if $\mathfrak{a}=\left(\mathfrak{m}_{1}\right)^{2}, D=R[1] / \mathfrak{a}$, then the notion of $D$-points is nothing but the notion of tangent vectors on $M$. We denote by $\tau=\pi\left(X_{1}\right)$, where $X_{1}$ is the indeterminate in $R[1]$ and $\pi: R[1] \rightarrow D$ is the natural projection.

Let $U$ be a coordinate neighborhood of $x_{0}$ in $M$ with coordinate system $\left\{x_{1}, \cdots, x_{n}\right\}$. Take a basis $\left\{1=B^{0}, B^{1}, \cdots, B^{N}\right\}$ of a local algebra $A$, where $B^{1}, \cdots, B^{N}$ span the maximal ideal $\mathfrak{m}$ of $A$. We define $x_{i, \lambda}: \pi_{A}^{-1}(U) \rightarrow R$ by

$$
\sum_{\lambda=0}^{N} x_{i, \lambda}\left(x^{\prime}\right) B^{\lambda}=x^{\prime}\left(x_{i}\right)
$$

for any $x^{\prime} \in \pi_{A}^{-1}(U)$, where we have used Remark 1.3 for $f=x_{i}$ ( $i=1$, $\cdots, n)$. We see readily that the set $M^{A}$ becomes a differentiable manifold of dimension $n(N+1)$ by the coordinate neighborhoods $\pi_{A}^{-1}(U)$ with coordinate system $\left\{x_{i, \lambda} \mid i=1, \cdots, n ; \lambda=0,1, \cdots, N\right\}$ induced by the coordinate system $\left\{x_{1}, \cdots, x_{n}\right\}$ on $U$. Clearly this differentiable structure on $M^{A}$ does not depend on the choice of the basis $\left\{B^{0}, \cdots, B^{N}\right\}$ of $A$.

Definition 1.5. The differentiable manifold $M^{A}$ defined above with the projection $\pi_{A}: M^{A} \rightarrow M$ will be called the bundle of $A$-points of $M$ (or bundle of infinitely near points of $M$ of $A$-kind).

Remark 1.6. The notion of bundle of $D$-kind is the same as that of tangent bundles. A tangent vector $X \in T_{x} M$ at $x$ is identified with $x^{\prime} \in M_{x}^{D}$ defined by $x^{\prime}(f)=f(x)+(X f) \cdot \tau$ for $f \in C^{\infty}(M)$.

Let $\Phi: M \rightarrow M^{\prime}$ be a map of a manifold $M$ into a manifold $M^{\prime}$. Then the map $\Phi^{A}: M^{A} \rightarrow M^{\prime A}$ is defined by

$$
\left(\Phi^{A}\left(x^{\prime}\right)\right) g=x^{\prime}(g \circ \Phi)
$$

for $x^{\prime} \in M^{A}$ and $g \in C^{\infty}\left(M^{\prime}\right)$. Clearly $\Phi^{A}$ is differentiable.

Lemma 1.7. Let $\pi_{i}: M_{1} \times M_{2} \rightarrow M_{i}(i=1,2)$ be the projections. Then $\left(M_{1} \times M_{2}\right)^{A}$ can be identified with $M_{1}^{A} \times M_{2}^{A}$ by the following identification

$$
x^{\prime}=\left(\pi_{1}^{A}\left(x^{\prime}\right), \pi_{2}^{A}\left(x^{\prime}\right)\right)
$$

for $x^{\prime} \in\left(M_{1} \times M_{2}\right)^{A}$.

Proof. Straightforward verification.

Lemma 1.8. Let $\Phi_{1}: M_{1} \rightarrow M_{1}^{\prime}, \Phi_{2}: M_{2} \rightarrow M_{2}^{\prime}, \Psi_{1}: M_{1} \rightarrow M_{1}^{\prime \prime}$ and $\Phi_{1}^{\prime}: M_{1}^{\prime}$ $\rightarrow M$ be differentiable maps, $M_{1}, M_{1}^{\prime}, M_{2}, M_{2}^{\prime}, M_{1}^{\prime \prime}, M$ being manifolds. Then we have the following equalities:

$$
\begin{array}{ll}
\left(\Phi_{1}^{\prime} \circ \Phi_{1}\right)^{A}=\Phi_{1}^{\prime A} \circ \Phi_{1}^{A}, & \left(\Phi_{1} \times \Phi_{2}\right)^{A}=\Phi_{1}^{A} \times \Phi_{2}^{A}, \\
\left(\Phi_{1}, \Psi_{1}\right)^{A}=\left(\Phi_{1}^{A}, \Psi_{2}^{A}\right), & \left(1_{M}\right)^{A}=1_{M^{A}},
\end{array}
$$


where $1_{M}$ stands for the identity map of $M$. Further, if we denote by $\pi_{i}$ (resp. $\left.\tilde{\pi}_{i}\right)$ the projection of $M_{1} \times M_{2}\left(\right.$ resp. $\left.M_{1}^{A} \times M_{2}^{A}\right)$ onto $M_{i}\left(\right.$ resp. $\left.M_{i}^{A}\right)$ for $i=$ 1,2 , then we have $\pi_{i}^{A}=\tilde{\pi}_{i}(i=1,2)$.

Proof. Straightforward verification by using (1.3) and (1.4).

Lemma 1.9. $\boldsymbol{R}^{A}$ can be identified with $A$ by $\boldsymbol{R}^{A} \ni x^{\prime} \rightarrow x^{\prime}(t) \in A$, where $t$ is the natural coordinates on $\boldsymbol{R}$.

Proof. Straightforward verification (cf. [7]).

Lemma 1.10. Let $A$ and $B$ be two local algebras. Then we can identify $A^{B}$ with $A \otimes B$ (cf. [7]).

Lemma 1.11. $A, B$ being as above, we can define canonically a diffeomorphism $\psi:\left(M^{A}\right)^{B} \rightarrow M^{A \otimes B}$.

Proof. Take $x^{\prime \prime} \in\left(M^{A}\right)^{B}$ and $f \in C^{\infty}(M)$. Since $f: M \rightarrow R$ is a $C^{\infty}$-map, we can consider the map $f^{A}: M^{A} \rightarrow \boldsymbol{R}^{A}=A$ (cf. Lemma 1.9). Hence using the map $\left(f^{A}\right)^{B}:\left(M^{A}\right)^{B} \rightarrow A^{B}=A \otimes B$ we can consider the map $x^{\prime}: C^{\infty}(M) \rightarrow A$ $\otimes B$ defined by $x^{\prime}(f)=\left(f^{A}\right)^{B}\left(x^{\prime \prime}\right) \in A \otimes B$, which is easily seen to be an $A \otimes B$ point on $M$. Thus we get a map $x^{\prime \prime} \rightarrow x^{\prime}$ from $\left(M^{A}\right)^{B}$ to $M^{A \otimes B}$, which can be verified to be a diffeomorphism (for detail, see [7]).

Corollary 1.12. $A$ and $B$ being as above, we can identify $x^{\prime \prime} \in\left(M^{A}\right)^{B}$ with $x_{1}^{\prime \prime} \in\left(M^{B}\right)^{A}$ for elements $x^{\prime \prime}$ and $x_{1}^{\prime \prime}$ characterized by

$$
\left(f^{A}\right)^{B}\left(x^{\prime \prime}\right)=\left(f^{B}\right)^{A}\left(x_{1}^{\prime \prime}\right)
$$

for every $f \in C^{\infty}(M)$, where we have identified $A \otimes B$ with $B \otimes A$.

Proof. Clear from the proof of Lemma 1.11.

Lemma 1.13. Let $G$ be a Lie group with group multiplication $\mu$. Then $G^{A}$ becomes a Lie group with group multiplication $\mu^{A}:(G \times G)^{A}=G^{A} \times G^{A}$ $\rightarrow G^{A}$.

Proof. Omitted (cf. [7]).

\section{2. $A$-module structures on the tangent spaces of $M^{A}$}

In this section, we define canonically an $A$-module structure on the tangent space of $M^{A}$ at every point of $M^{A}$.

Let $\mu: R \times M^{D} \rightarrow M^{D}$ be the scalar multiplication of the tangent vectors of $M$, i.e., $\mu(t, X)=t \cdot X$ for $t \in R, X \in M^{D}$. Since $R^{A}=A$ and $\left(M^{D}\right)^{A}=$ $\left(M^{A}\right)^{D}$ by our identification (cf. Corollary 1.12), the map $\mu^{A}:\left(R \times M^{D}\right)^{A}=$ $\boldsymbol{R}^{A} \times\left(M^{D}\right)^{A} \rightarrow\left(M^{D}\right)^{A}$ can be considered as the map $\mu^{A}: A \times\left(M^{A}\right)^{D} \rightarrow\left(M^{A}\right)^{D}$.

Definition 2.1. Put $\mu^{A}\left(a, x^{\prime \prime}\right)=a \cdot x^{\prime \prime}$ for $a \in A, x^{\prime \prime} \in\left(M^{A}\right)^{D}$. We denote by $\pi_{D}$ (resp. $\tilde{\pi}_{D}$ ) the projection $M^{D} \rightarrow M\left(\right.$ resp. $\left.\left(M^{A}\right)^{D} \rightarrow M^{A}\right)$.

Lemma 2.2. The notation being as above, we have

(i) $\tilde{\pi}_{D}\left(a \cdot x^{\prime \prime}\right)=\tilde{\pi}_{D}\left(x^{\prime \prime}\right)$ for every $a \in A$ and $x^{\prime \prime} \in\left(M^{A}\right)^{D}$,

(ii) for any $x^{\prime} \in M^{A}$, the tangent space $\left(M^{A}\right)_{x^{\prime}}^{D}$ becomes an $A$-module by the multiplication $\left(a, x^{\prime \prime}\right) \rightarrow a \cdot x^{\prime \prime}$ for $\left(a, x^{\prime \prime}\right) \in A \times\left(M^{A}\right)_{x^{\prime}}^{D}$

Remark 2.3. In fact, in the next section (cf. Corollary 3.10) we shall show 
that $\left(M^{A}\right)_{x^{\prime}}^{D}$ is a free $A$-module for any $x^{\prime} \in M^{A}$.

Proof of Lemma 2.2. (i) Consider the following diagram:

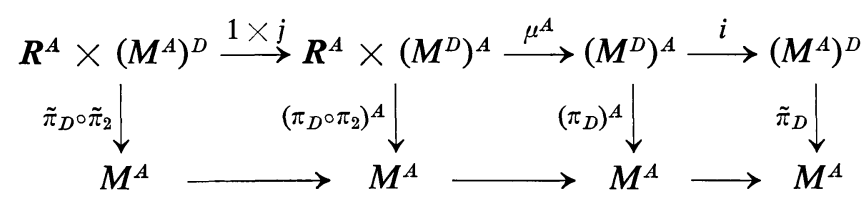

where $\tilde{\pi}_{2}: \boldsymbol{R}^{A} \times\left(M^{A}\right)^{D} \rightarrow\left(M^{A}\right)^{D}$ (resp. $\pi_{2}: R \times M^{D} \rightarrow M^{D}$ ) is the projection and $j^{-1}=i:\left(M^{D}\right)^{A} \rightarrow\left(M^{A}\right)^{D}$ is the identification map (cf. Corollary 1.12). Since $\pi_{D} \circ \mu=\pi_{D} \circ \pi_{2}$, the middle rectangle of (2.1) is commutative. It is now sufficient to verify the commutativity of the right rectangle of (2.1), because $a \cdot x^{\prime \prime}=\left(i \circ \mu^{A} \circ(1 \times j)\right)\left(a, x^{\prime \prime}\right)$ for $\left(a, x^{\prime \prime}\right) \in A \times\left(M^{A}\right)^{D}$, and the commutativity of the left rectangle is implied by that of the right one.

Take $x^{\prime \prime} \in\left(M^{D}\right)^{A}$ and put $x_{1}^{\prime \prime}=i\left(x^{\prime \prime}\right) \in\left(M^{A}\right)^{D}$. Then for any $f \in C^{\infty}(M)$, we have

$$
\left(f^{D}\right)^{A}\left(x^{\prime \prime}\right)=\left(f^{A}\right)^{D}\left(x_{1}^{\prime \prime}\right)
$$

(cf. Corollary 1.12), where we have identified: $A \otimes D=D \otimes A$. To show that $\left(\pi_{D}\right)^{A}\left(x^{\prime \prime}\right)=\tilde{\pi}_{D}\left(x_{1}^{\prime \prime}\right)$, it suffices to show

$$
\left(\left(\pi_{D}\right)^{A}\left(x^{\prime \prime}\right)\right)(f)=\left(\tilde{\pi}_{D}\left(x_{1}^{\prime \prime}\right)\right)(f)
$$

for $f \in C^{\infty}\left(M^{A}\right)$. (2.3) is equivalent to

$$
x^{\prime \prime}\left(f \circ \pi_{D}\right)=f^{A}\left(\tilde{\pi}_{D}\left(x_{1}^{\prime \prime}\right)\right) .
$$

Now, we know that $f^{D} \equiv f \circ \pi_{D}(\bmod \boldsymbol{R} \cdot \tau)$, where $D=\boldsymbol{R} \oplus \boldsymbol{R} \tau$. Therefore we have $\left(f^{A}\right)^{D} \equiv f^{A} \circ \tilde{\pi}_{D}(\bmod A \otimes \boldsymbol{R} \tau)$. Considering the $A$-components of (2.2) in $A \otimes D=A \oplus A \otimes R \tau$, we obtain (2.4), since $\left(f^{D}\right)^{A}\left(x^{\prime \prime}\right)=x^{\prime \prime}\left(f^{D}\right) \equiv$ $x^{\prime \prime}\left(f \circ \pi_{D}\right)(\bmod A \otimes R \cdot \tau)$. Thus (i) is proved.

(ii) Let $\mu_{0}: R \times R \rightarrow R$ and $\mu_{A}: A \times A \rightarrow A$ be the multiplication in $R$ and $A$ respectively. We see easily that $\left(\mu_{0}\right)^{A}=\mu_{A}$. The equality $(t \cdot s) \cdot X=t \cdot(s \cdot X)$ for $t, s \in R$ and $X \in M^{D}$ can be written as $\mu \circ\left(\mu_{0} \circ \pi_{12}, \pi_{3}\right)=\mu \circ\left(\pi_{1}, \mu \circ \pi_{23}\right)$, where $\pi_{12}: R \times R \times M \rightarrow R \times R, \pi_{3}: R \times R \times M \rightarrow M$ etc. denote the natural projection. Then by the functoriality of $\mu \rightarrow \mu^{A}$, etc. (cf. Lemma 1.8) it follows that $\mu^{A} \circ\left(\mu_{A} \circ \tilde{\pi}_{12}, \tilde{\pi}_{3}\right)=\mu^{A} \circ\left(\tilde{\pi}_{1}, \mu^{A} \circ \tilde{\pi}_{23}\right)$, where $\tilde{\pi}_{12}: A \times A \times M^{A}$ $\rightarrow A \times A$ etc. denote the natural projection similar to $\pi_{12}$ etc. Thus we get the associativity: $(a \cdot b) \cdot x^{\prime \prime}=a \cdot\left(b \cdot x^{\prime \prime}\right)$ for $a, b \in A, x^{\prime \prime} \in M^{A}$.

The distributivity $(a+b) \cdot x^{\prime \prime}=a \cdot x^{\prime \prime}+b \cdot x^{\prime \prime}, a \cdot\left(x^{\prime}+x^{\prime \prime}\right)=a \cdot x^{\prime}+a \cdot x^{\prime \prime}$ are similarly proved by using the addition $\alpha: M^{D} \oplus M^{D} \rightarrow M^{D}$ of tangent vectors, where $M^{D} \oplus M^{D}$ denotes the Whitney sum of the tangent bundles $M^{D}$ with itself. 
Remark 2.4. We can prove (ii) of Lemma 2.1 more quickly by using the local coordinate system around $x^{\prime \prime} \in\left(M^{D}\right)^{A}$ and the local expression of $\mu^{A}$ by coordinates. In fact, taking a coordinate system $\left\{x_{1}, \cdots, x_{n}\right\}$ around $x$, we see that

$$
x_{i, \varepsilon, \lambda}\left(a \cdot x^{\prime \prime}\right)= \begin{cases}\sum a_{\kappa} x_{i, \varepsilon, \nu}\left(x^{\prime \prime}\right) C_{\lambda}^{\kappa, \nu} & (\varepsilon=1), \\ x_{i, \varepsilon, \lambda}\left(x^{\prime \prime}\right) & (\varepsilon=0)\end{cases}
$$

for $a=\sum a_{\kappa} B^{\kappa}, B^{x} \cdot B^{\nu}=\sum C_{\lambda}^{\kappa, \nu} B^{\lambda}$ (cf. (1.2)).

We want to give another interpretation of the $A$-module structure on the tangent space $T_{x^{\prime}}\left(M^{A}\right)$ with $x^{\prime} \in M^{A}$. Let $L \in T_{x^{\prime}}\left(M^{A}\right)$ be a tangent vector at $x^{\prime} \in M^{A}$. Then there exists a curve $t \rightarrow x_{t}^{\prime}$ on $M^{A}$ such that $x_{0}^{\prime}=x^{\prime}$ and that

$$
L \tilde{f}=\left.\frac{d \tilde{f}\left(x_{t}^{\prime}\right)}{d t}\right|_{t=0}
$$

for $\tilde{f} \in C^{\infty}\left(M^{A}\right)$. We define $L^{\prime}: C^{\infty}(M) \rightarrow A$ by

$$
L^{\prime} f=\left.\frac{d\left(x_{t}^{\prime}(f)\right)}{d t}\right|_{t=0} .
$$

Lemma 2.5. The map $L^{\prime}: C^{\infty}(M) \rightarrow A$ is well-defined and linear, and has the property

$$
L^{\prime}(f \cdot g)=L^{\prime} f \cdot x^{\prime}(g)+x^{\prime}(f) \cdot L^{\prime} g
$$

for $f, g \in C^{\infty}(M)$.

Proof. Since $x_{t}^{\prime}(f)=f^{A}\left(x_{t}^{\prime}\right), x_{t}^{\prime}(f)$ is differentiable with respect to $t$ and $\left.\frac{d x_{t}^{\prime}(f)}{d t}\right|_{t=0}=L\left(f^{A}\right)$, (cf. (2.5)). If another curve $x_{t}^{\prime \prime}$ on $M^{A}$ satisfies $x_{0}^{\prime \prime}=x^{\prime}$ and $L \tilde{f}=\left.\frac{d \tilde{f}\left(x_{t}^{\prime \prime}\right)}{d t}\right|_{t=0}$, then we have $\left.\frac{d x_{t}^{\prime}(f)}{d t}\right|_{t=0}=\left.\frac{d x_{t}^{\prime \prime}(f)}{d t}\right|_{t=0}$. Thus $L^{\prime}$ is welldefined.

(2.7) can be verified directly.

Definition 2.6. We denote by $T_{x^{\prime}}^{\prime}\left(M^{A}\right)$ the set of all linear map $L^{\prime}: C^{\infty}(M)$ $\rightarrow A$ such that (2.7) holds for every $f, g \in C^{\infty}(M)$.

Remark 2.7. For $L^{\prime} \in T_{x^{\prime}}^{\prime} M^{A}$, we can define $L^{\prime} h$ for any $C^{\infty}$-function $h$ around $x$.

Thus we have obtained a map $j: T_{x^{\prime}} M^{A} \rightarrow T_{x^{\prime}}^{\prime} M^{A}$ by $j(L)=L^{\prime}$, (cf. (2.6)).

Lemma 2.8. The map $j$ is a bijective linear map.

Proof. Let $L_{1}, L_{2} \in T_{x^{\prime}} M^{A}$. For $f \in C^{\infty}(M)$ we have $\left(L_{1}+L_{2}\right)^{\prime} f=\left(L_{1}+L_{2}\right) f^{A}$ $=L_{1} f^{A}+L_{2} f^{A}=L_{1}^{\prime} f+L_{2}^{\prime} f=\left(L_{1}^{\prime}+L_{2}^{\prime}\right) f$. Similarly $\left(\alpha L_{1}\right)^{\prime} f=\left(\alpha L_{1}\right) f^{A}=\alpha\left(L_{1} f^{A}\right)$ $=\alpha\left(L_{1}^{\prime} f\right)=\left(\alpha L_{1}^{\prime}\right) f$ for $\alpha \in \boldsymbol{R}$. Thus $j$ is linear.

To prove the bijectivity of $j$, we first prove 


$$
\operatorname{dim} T_{x^{\prime}}^{\prime} M^{A} \leq \operatorname{dim} M^{A} .
$$

In fact, take a coordinate system $\left\{x_{1}, \cdots, x_{n}\right\}$ around $x$, and consider the linear map $g: T_{x^{\prime}}^{\prime} M^{A} \rightarrow A^{n}$ by $g\left(L^{\prime}\right)=\left(L^{\prime} x_{1}, \cdots, L^{\prime} x_{n}\right)$. We show first that $g$ is injective. Take $L_{1}^{\prime}$ and $L_{2}^{\prime} \in T_{x^{\prime}}^{\prime} M^{A}$ and assume $L_{1}^{\prime} x_{i}=L_{2}^{\prime} x_{i}$ for every $i=$ $1, \cdots, n$. For any $f \in C^{\infty}(M)$ we can find a polynomials $P, Q$ of $x_{1}, \cdots, x_{n}$ and $g \in C^{\infty}(M)$ such that

$$
f=P+g \cdot Q
$$

holds on some neighborhood of $x$, where $Q$ is homogeneous and of degree $\geq$ height of $A$. Then we have

$$
\begin{aligned}
L_{1}^{\prime} f & =L_{1}^{\prime}(P)+L_{1}^{\prime}(g) x^{\prime}(Q)+x^{\prime}(g) L_{1}^{\prime}(Q) \\
& =L_{1}^{\prime}(P)+x^{\prime}(g) L_{1}^{\prime}(Q)=L_{2}^{\prime}(P)+x^{\prime}(g) L_{2}^{\prime}(Q)=L_{2}^{\prime} f,
\end{aligned}
$$

where we have used the fact that $x^{\prime}\left(f_{1} \cdots f_{h}\right)=0$ for $f_{i} \in C^{\infty}(M)$ with $f_{i}(x)$ $=0$. Thus $L_{1}^{\prime}=L_{2}^{\prime}$, which proves the injectivity of $g$. Therefore we get (2.7).

To prove the injectivity of $j$, it suffices to show that $L^{\prime} f=0$ for every $f \in$ $C^{\infty}(M)$ implies $L=0$. Now $\sum L\left(x_{i, \lambda}\right) \cdot B^{\lambda}=L\left(x_{i}{ }^{A}\right)=L^{\prime} x_{i}=0$, which implies $L\left(x_{i, \lambda}\right)=0$ for any $i=1, \cdots, n ; \lambda=0, \cdots, N$. Thus $L=0$. The injectivity and the inequality (2.7) imply the bijectivity of $j$.

Remark 2.9. $T_{x^{\prime}}^{\prime} M^{A}$ becomes canonically an $A$-module, i.e., for $a \in A$ and $L^{\prime} \in T_{x^{\prime}}^{\prime}\left(M^{A}\right)$ we define $a \cdot L^{\prime} \in T_{x^{\prime}}^{\prime}\left(M^{A}\right)$ by

$$
\left(a \cdot L^{\prime}\right) f=a \cdot\left(L^{\prime} f\right)
$$

for $f \in C^{\infty}(M)$.

Lemma 2.10. For any $a \in A$ and $L \in T_{x^{\prime}}\left(M^{A}\right)$, we have

$$
(a \cdot L)^{\prime}=a \cdot L^{\prime} .
$$

(Cf. Definition 2.1 for $a \cdot L$ ).

Proof. To make the several identifications more clear, we introduce the following notation. For $L \in T_{x^{\prime}} M^{A}$ the identified element in $\left(M^{A}\right)_{x^{\prime}}^{D}$ will be denoted by $L^{*}$, and conversely for $K \in\left(M^{A}\right)_{x^{\prime}}^{D}$ the corresponding element in $T_{x^{\prime}} M^{A}$ will be denoted by ${ }^{*} K$. Similarly for $S \in T_{x^{\prime}}^{\prime} M^{A}$, we denote ${ }^{\prime} S=j^{-1}(S)$. Further for $L^{*} \in\left(M^{A}\right)^{D}$ the corresponding element in $\left(M^{D}\right)^{A}$ will be denoted by $L_{1}^{*}$. Then (2.8) means more precisely

$$
\left({ }^{*}\left(a \cdot L^{*}\right)\right)^{\prime}=a \cdot L^{\prime}
$$

Now $(2.8)^{\prime}$ is equivalent to

$$
\mu^{A}\left(a, L_{1}^{*}\right)=\left({ }^{\prime}\left(a L^{\prime}\right)\right)_{1}^{*},
$$


which is equivalent to

$$
\left(f^{D}\right)^{A}\left(\mu^{A}\left(a, L_{1}^{*}\right)\right)=\left(f^{A}\right)^{D}\left(\left({ }^{\prime}\left(a L^{\prime}\right)\right)^{*}\right)
$$

for $f \in C^{\infty}(M)$.

The left hand side of $(2.8)^{\prime \prime \prime}$ is equal to

$$
\left(f^{D} \circ \mu\right)^{A}\left(a, L_{1}^{*}\right)=\left(a, L_{1}^{*}\right)\left(f^{D} \circ \mu\right),
$$

while the right hand side of $(2.8)^{\prime \prime \prime}$ is equal to

$$
\begin{aligned}
\left({ }^{\prime}\left(a L^{\prime}\right)\right)^{*}\left(f^{A}\right) & =f^{A}\left(x^{\prime}\right)+{ }^{\prime}\left(a L^{\prime}\right) f^{A} \cdot \tau \\
& =f^{A}\left(x^{\prime}\right)+\left(a \cdot L^{\prime}\right) f \cdot \tau=f^{A}\left(x^{\prime}\right)+a \cdot L^{\prime} f \cdot \tau .
\end{aligned}
$$

Therefore it remains to verify

$$
\left(a, L_{1}^{*}\right)\left(f^{D} \circ \mu\right)=f^{A}\left(x^{\prime}\right)+a \cdot L^{\prime} f \cdot \tau
$$

for $f \in C^{\infty}(M)$.

Now, since $L^{*}$ and $L_{1}^{*}$ are corresponding elements in $\left(M^{A}\right)^{D}$ and $\left(M^{D}\right)^{A}$, we have

$$
\left(f^{D}\right)^{A}\left(L_{1}^{*}\right)=\left(f^{A}\right)^{D}\left(L^{*}\right)
$$

for $f \in C^{\infty}(M)$.

Put $K=\left(a, L_{1}^{*}\right)$. Then we have $K\left(g \circ \pi_{1}\right)=a(g), K\left(g^{\prime} \circ \pi_{2}\right)=L_{1}^{*}\left(g^{\prime}\right)$ for $g \in C^{\infty}(R), g^{\prime} \in C^{\infty}\left(M^{D}\right)$. Next, we have, for $(t, X) \in \boldsymbol{R} \times M^{D}$,

$$
\begin{aligned}
\left(f^{D} \circ \mu\right)(t, X) & =f^{D}(t X)=f(\pi X)+(t X) f \cdot \tau \\
& =(f \circ \pi) \circ \pi_{2}(t, X)+\left(1 \circ \pi_{1}(t, X)\right) \cdot \pi_{2}(t, X) f \cdot \tau \\
& =(f \circ \pi) \circ \pi_{2}(t, X)+\left(1 \circ \pi_{1}\right) \cdot\left(f^{\prime} \circ \pi_{2}\right)(t, X) \cdot \tau,
\end{aligned}
$$

where $f^{\prime} \in C^{\infty}\left(M^{D}\right)$ is defined by $f^{\prime}(X)=X f$ for $X \in M^{D}$. Hence we have

$$
\begin{aligned}
K\left(f^{D} \circ \mu\right) & =K\left((f \circ \pi) \circ \pi_{2}\right)+K\left(1 \circ \pi_{1}\right) \cdot K\left(f^{\prime} \circ \pi_{2}\right) \cdot \tau \\
& =L_{1}^{*}(f \circ \pi)+a \cdot L_{1}^{*} f^{\prime} \cdot \tau .
\end{aligned}
$$

On the other hand, from (2.10) we get

$$
\begin{aligned}
& \left(f^{D}\right)^{A}\left(L_{1}^{*}\right)=L_{1}^{*}\left(f^{D}\right)=L_{1}^{*}\left(f \circ \pi+f^{\prime} \cdot \tau\right)=L_{1}^{*}(f \circ \pi)+L_{1}^{*} f^{\prime} \cdot \tau, \\
& \left(f^{A}\right)^{D}\left(L^{*}\right)=L^{*}\left(f^{A}\right)=f^{A}\left(x^{\prime}\right)+L f^{A} \cdot \tau=f^{A}\left(x^{\prime}\right)+L^{\prime} f \cdot \tau,
\end{aligned}
$$

which imply

$$
L_{1}^{*}(f \circ \pi)=f^{A}\left(x^{\prime}\right), \quad L_{1}^{*} f^{\prime}=L^{\prime} f .
$$

Combining (2.10), (2.11) and (2.12), we get (2.9). 


\section{Lifting of vector fields}

We denote by $\mathscr{T}_{0}^{1}(M)$ the set of all vector fields on $M$. Take $X \in \mathscr{T}_{0}^{1}(M)$. The corresponding $X^{\prime}: M \rightarrow M^{D}$ is defined by

$$
X^{\prime}(x) f=f(x)+(X(x) f) \cdot \tau \in D
$$

for $f \in C^{\infty}(M)$ and $x \in M$. The map $X^{\prime}$ induces a map $X^{A}: M^{A} \rightarrow\left(M^{D}\right)^{A}$. Consider the map $\tilde{X}=i \circ X^{A}: M^{A} \rightarrow\left(M^{A}\right)^{D}$, where $i:\left(M^{D}\right)^{A} \rightarrow\left(M^{A}\right)^{D}$ is the identification map. The commutativity of the right triangle of the diagram (2.1) implies that $\tilde{X}\left(x^{\prime}\right) \in\left(M^{A}\right)_{x^{\prime}}^{D}$ for every $x^{\prime} \in M^{A}$. Hence by Remark 1.6 we obtain a tangent vector in $T_{x^{\prime}}\left(M^{A}\right)$ corresponding to $\tilde{X}\left(x^{\prime}\right)$, which we denote by $X^{A}\left(x^{\prime}\right)$.

Thus we obtain a vector field $X^{A} \in \mathscr{T}_{0}^{1}\left(M^{A}\right)$.

Definition 3.1. The vector field $X^{A}$ is called the lift of $X$ to $M^{A}$.

Remark 3.2. Any $X \in \mathscr{T}_{0}^{1}\left(M^{A}\right)$ can be extended to a derivation of $C^{\infty}\left(M^{A}, A\right)$ by

$$
X \tilde{f}=X\left(\sum f_{i} B^{i}\right)=\sum\left(X f_{i}\right) \cdot B^{i}
$$

where $\left\{1, B^{1}, \cdots, B^{N}\right\}$ is a basis of $A$, and $\tilde{f}=\sum f_{i} B^{i}$ with $f_{i} \in C^{\infty}\left(M^{A}\right)$.

Lemma 3.3. For any $X \in \mathscr{T}_{0}^{1}(M)$ and $f \in C^{\infty}(M)$, we have

$$
(X f)^{A}=X^{A} f^{A} \text {. }
$$

Proof. We have to show

$$
(X f)^{A}\left(x^{\prime}\right)=\left(X^{A} f^{A}\right)\left(x^{\prime}\right)
$$

for $x^{\prime} \in M^{A}$. Put $x^{\prime \prime}=X^{A}\left(x^{\prime}\right) \in\left(M^{D}\right)^{A}$. Then $x_{1}^{\prime \prime}=\left(X^{A}\left(x^{\prime}\right)\right)^{\prime}$ is the element corresponding to $x^{\prime \prime}$ in $\left(M^{A}\right)^{D}$ (for the notation ( $)^{\prime}$ see Remark 1.6). Using Corollary 1.12 we have

$$
\begin{aligned}
\left(f^{D}\right)^{A}\left(x^{\prime \prime}\right) & =\left(f^{A}\right)^{D}\left(x_{1}^{\prime \prime}\right)=x_{1}^{\prime \prime}\left(f^{A}\right)=\left(X^{A}\left(x^{\prime}\right)\right)^{\prime} f^{A} \\
& =f^{A}\left(x^{\prime}\right)+X^{A}\left(x^{\prime}\right) f^{A} \cdot \tau .
\end{aligned}
$$

The left hand side of (3.3) is equal to

$$
\left(\left(f^{D}\right)^{A} \circ X^{\prime A}\right)\left(x^{\prime}\right)=\left(f^{D} \circ X^{\prime}\right)^{A}\left(x^{\prime}\right)=x^{\prime}\left(f^{D} \circ X^{\prime}\right) .
$$

Since

$$
\begin{aligned}
\left(f^{D} \circ X^{\prime}\right)(x) & =f^{D}\left(X^{\prime}(x)\right)=X^{\prime}(x)(f)=f(x)+(X f)(x) \cdot \tau \\
& =(f+X f \cdot \tau)(x)
\end{aligned}
$$

(3.5) is equal to

$$
x^{\prime}(f)+x^{\prime}(X f) \cdot \tau=f^{A}\left(x^{\prime}\right)+(X f)^{A}\left(x^{\prime}\right) \cdot \tau .
$$


Comparing (3.4) and (3.6), we obtain (3.3).

Lemma 3.4. The map $X \rightarrow X^{A}$ is linear.

Proof. Take $f \in C^{\infty}(M)$ and $X, Y \in \mathscr{T}_{0}^{1}(M)$. Then by Lemma 3.3 we have $(X+Y)^{A} f^{A}=((X+Y) f)^{A}=(X f+Y f)^{A}=(X f)^{A}+(Y f)^{A}=X^{A} f^{A}+Y^{A} f^{A}$ $=\left(X^{A}+Y^{A}\right) f^{A}$, and therefore $\left((X+Y)^{A}\right)^{\prime} f=(X+Y)^{A} f^{A}=\left(X^{A}+Y^{A}\right) f^{A}$ $=\left(X^{A}+Y^{A}\right)^{\prime} f$, which implies $\left((X+Y)^{A}\right)^{\prime}=\left(X^{A}+Y^{A}\right)^{\prime}$ (for the notation ()$^{\prime}$ see Lemme 2.8). Hence we get $(X+Y)^{A}=X^{A}+Y^{A}$. Similarly, $(\alpha \cdot X)^{A}=\alpha \cdot X^{A}$ for $\alpha \in R$.

Lemma 3.5. For any $f \in C^{\infty}(M)$ and $X \in \mathscr{T}_{0}^{1}(M)$, we have

$$
(f \cdot X)^{A}=f^{A} \cdot X^{A}
$$

equivalently,

$$
(f X)^{A}\left(x^{\prime}\right)=f^{A}\left(x^{\prime}\right) X^{A}\left(x^{\prime}\right)
$$

for every $x^{\prime} \in M^{A}$ (cf. Definition 2.1).

Proof. Let $\mu: R \times M^{D} \rightarrow M^{D}$ be the scalar multiplication of tangent vectors. Identifying $X$ with its corresponding $X^{\prime}: M \rightarrow M^{D}$ (cf. Definition 3.1), we have

$$
(f \cdot X)^{A}=(\mu \circ(f, X))^{A}=\mu^{A} \circ(f, X)^{A}=\mu^{A} \circ\left(f^{A}, X^{A}\right)=f^{A} \cdot X^{A} .
$$

Definition 3.6. For $\tilde{X} \in \mathscr{T}_{0}^{1}\left(M^{A}\right)$, we define a map $\tilde{X}^{\prime}: C^{\infty}(M) \rightarrow C^{\infty}\left(M^{A}, A\right)$ by

$$
\left(\tilde{X}^{\prime} f\right)\left(x^{\prime}\right)=\left(\tilde{X}\left(x^{\prime}\right)\right)^{\prime} f=\left(j\left(\tilde{X}\left(x^{\prime}\right)\right)\right) f
$$

for $f \in C^{\infty}(M)$ and $x^{\prime} \in M^{A}$ (cf. Remark 2.7).

Remark 3.7. By Lemma 2.8, we have

$$
(g \cdot \tilde{X})^{\prime}=g \cdot \tilde{X}^{\prime}
$$

for $g \in C^{\infty}\left(M^{A}, A\right), \tilde{X} \in \mathscr{T}_{0}^{1}\left(M^{A}\right)$.

Lemma 3.8. For $a \in A, X \in \mathscr{T}_{0}^{1}(M)$ and $f \in C^{\infty}(M)$, we have

$$
\left(a \cdot X^{A}\right) f^{A}=a \cdot(X f)^{A} .
$$

Proof. We have

$$
\left(a \cdot X^{A}\right) f^{A}=\left(a \cdot X^{A}\right)^{\prime} f=a \cdot\left(\left(X^{A}\right)^{\prime} f\right)=a \cdot\left(X^{A} f^{A}\right)=a \cdot(X f)^{A},
$$

where we have used Lemma 2.5, Lemma 3.3 and Remark 3.7.

Lemma 3.9. Let $\left\{x_{1}, \cdots, x_{n}\right\}$ be a coordinate system on some neighborhood of $M$. Then we have

$$
B^{\lambda} \cdot\left(\partial / \partial x_{i}\right)^{A}=\partial / \partial x_{i, \lambda}
$$


for $i=1, \cdots, n ; \lambda=0, \cdots, N$.

Proof. We have $\left(B^{\lambda}\left(\partial / \partial x_{i}\right)^{A}\right)\left(x_{j}\right)^{A}=B^{\lambda}\left(\partial x_{j} / \partial x_{i}\right)^{A}=B^{\lambda}\left(\delta_{i j}\right)^{A}=B^{\lambda} \cdot \delta_{i j}$. On the other hand we have

$$
\left(\partial / \partial x_{i, 2}\right)\left(x_{j}\right)^{A}=\left(\partial / \partial x_{i, 2}\right)\left(\sum_{\nu} x_{j, \nu} B^{\nu}\right)=\delta_{i j} B^{\lambda} .
$$

Hence we get (3.9).

Corollary 3.10. For any $x^{\prime} \in M^{A}$, the $A$-module $T_{x^{\prime}} M^{A}$, is a free $A$-module. Proof. Take $X_{i}=\left(\partial / \partial x_{i}\right)_{x^{\prime}}^{A}(i=1, \cdots, n)$. Then $\left\{X_{1}, \cdots, X_{n}\right\}$ is a free $A$-basis of $T_{x^{\prime}} M^{A}$.

Lemma 3.11. For any $X, Y \in \mathscr{T}_{0}^{1}(M)$ we have

$$
\left[X^{A}, Y^{A}\right]=[X, Y]^{A} .
$$

Proof. For any $f \in C^{\infty}(M)$, we have

$$
\begin{aligned}
{\left[X^{A}, Y^{A}\right] f^{A} } & =X^{A} Y^{A} f^{A}-Y^{A} X^{A} f^{A}=(X Y f-Y X f)^{A} \\
& =([X, Y] f)^{A}=[X, Y]^{A} f^{A} .
\end{aligned}
$$

Hence we have

$$
\left[X^{A}, Y^{A}\right]^{\prime} f=\left[X^{A}, Y^{A}\right] f^{A}=[X, Y]^{A} f^{A}=\left([X, Y]^{A}\right)^{\prime} f,
$$

which implies $\left[X^{A}, Y^{A}\right]^{\prime}=\left([X, Y]^{A}\right)^{\prime}$ and hence we get $\left[X^{A}, Y^{A}\right]=[X, Y]^{A}$.

Lemma 3.12. For any $a, b \in A$ and $X, Y \in \mathscr{T}_{0}^{1}(M)$ we have

$$
\left[a X^{A}, b Y^{A}\right]=(a \cdot b) \cdot[X, Y]^{A} .
$$

Proof. We calculate as follows : for any $f \in C^{\infty}(M)$

$$
\begin{aligned}
{\left[a X^{A}, b Y^{A}\right] f^{A} } & =\left(a X^{A}\right)\left(b Y^{A}\right) f^{A}-\left(b Y^{A}\right)\left(a X^{A}\right) f^{A} \\
& =\left(a \cdot X^{A}\right)\left(b \cdot(Y f)^{A}\right)-b Y^{A}\left(a \cdot(X f)^{A}\right) \\
& =b \cdot\left(a X^{A}(Y f)^{A}\right)-a \cdot\left(b Y^{A} \cdot(X f)^{A}\right) \\
& =b \cdot a \cdot(X Y f)^{A}-a \cdot b(Y X f)^{A} \\
& =(a \cdot b) \cdot([X, Y] f)^{A}=(a \cdot b)\left([X, Y]^{A} f^{A}\right) \\
& =\left((a b)[X, Y]^{A}\right) f^{A} .
\end{aligned}
$$

By the same argument as in Lemma 3.10 we get (3.10).

Remark 3.13. We can verify that if $\left\{\Phi^{t}\right\}$ is a one-parameter group of diffeomorphisms on $M$ generated by a vector field $X$, then the one-parameter group $\left\{\left(\Phi^{t}\right)^{A}\right\}$ induces the vector field $X^{A}$. 


\section{Lifting of covariant tensor fields}

Take $f \in C^{\infty}(M)$. Since $f^{A}: M^{A} \rightarrow A$ is an $A$-valued function, we can consider $d f^{A}: T\left(M^{A}\right) \rightarrow A$. On the other hand, since $d f: M^{D} \rightarrow R$ is a function, we can consider $(d f)^{A}:\left(M^{D}\right)^{A} \rightarrow \boldsymbol{R}^{A}=A$.

Lemma 4.1. Identifying $T\left(M^{A}\right)=\left(M^{A}\right)^{D}$ with $\left(M^{D}\right)^{A}$, we have

$$
(d f)^{A}=d f^{A} .
$$

Proof. Let $\pi$ (resp. $\tilde{\pi}$ ) be the projection $\pi: \boldsymbol{R}^{D}=\boldsymbol{R} \oplus \boldsymbol{R} \cdot \tau \rightarrow \boldsymbol{R} \cdot \tau=\boldsymbol{R}$ (resp. $\tilde{\pi}: A^{D}=A \oplus A \cdot \tau \rightarrow A \cdot \tau=A$ ). Then we have, by definition, $d f=$ $\pi \circ f^{D}, d f^{A}=\tilde{\pi} \circ\left(f^{A}\right)^{D}$. Hence $(d f)^{A}=\pi^{A} \circ\left(f^{D}\right)^{A}$. Then the commutative diagram

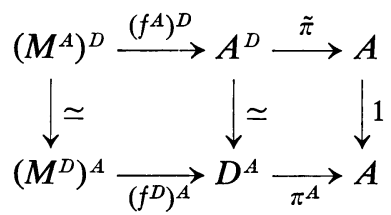

proves (4.1). q.e.d.

Take a 1-form $\theta \in \mathscr{T}_{1}^{0}(M)$. Then $\theta$ can be considered as a function $\theta: M^{D}$ $\rightarrow \boldsymbol{R}$. Hence $\theta^{A}:\left(M^{D}\right)^{A} \rightarrow A$ is an $A$-valued function on $\left(M^{D}\right)^{A}=\left(M^{A}\right)^{D}$. To prove that $\theta^{A}$ is in fact a 1 -form on $M^{A}$, we shall first prove

Lemma 4.2. Take $\theta_{1}, \theta_{2} \in \mathscr{T}_{1}^{0}(M)$. Then we have

$$
\left(\theta_{1}+\theta_{2}\right)^{A}=\theta_{1}^{A}+\theta_{2}{ }^{A} .
$$

Proof. Let $\alpha: \boldsymbol{R} \times \boldsymbol{R} \rightarrow \boldsymbol{R}$ (resp. $\alpha_{A}: A \times A \rightarrow A$ ) be the addition in $\boldsymbol{R}$ (resp. $A$ ). Then we know that $\alpha_{A}=\alpha^{A}$, and therefore that

$$
\begin{aligned}
\left(\theta_{1}+\theta_{2}\right)^{A} & =\left(\alpha \circ\left(\theta_{1}, \theta_{2}\right)\right)^{A}=\alpha^{A} \circ\left(\theta_{1}{ }^{A}, \theta_{2}{ }^{A}\right) \\
& =\alpha_{A}\left(\theta_{1}{ }^{A}, \theta_{2}{ }^{A}\right)=\theta_{1}{ }^{A}+{\theta_{2}}^{A} .
\end{aligned}
$$

Lemma 4.3. For $f \in C^{\infty}(M)$ and $\theta \in \mathscr{T}_{1}^{0}(M)$, we have

$$
(f \cdot \theta)^{A}=f^{A} \cdot \theta^{A} .
$$

Proof. Let $\mu_{0}: R \times R \rightarrow R$ (resp. $\mu_{A}: A \times A \rightarrow A$ ) be the multiplication in $\boldsymbol{R}$ (resp. in $A$ ). Then we know $\left(\mu_{0}\right)^{A}=\mu_{A}$, and therefore $(f \cdot \theta)^{A}=\left(\mu_{0} \circ(f, \theta)\right)^{A}$ $=\left(\mu_{0}\right)^{A} \circ\left(f^{A}, \theta^{A}\right)=\mu_{A} \circ\left(f^{A}, \theta^{A}\right)=f^{A} \cdot \theta^{A}$.

Lemma 4.4. For any $\theta \in \mathscr{T}_{1}^{0}(M)$, we have $\theta^{A} \in \mathscr{T}_{1}^{0}\left(M^{A}\right)$.

Proof. Since the problem is local, we can assume that $\theta=\sum g_{i} d f_{i}$ with $g_{i}, f_{i} \in C^{\infty}(M)$. By (4.1), (4.2), (4.3) we have $\theta^{A}=\sum g_{i}{ }^{A} d f_{i}{ }^{A}$, which is a 1 -form on $M^{A}$.

Lemma 4.5. For $\theta \in \mathscr{T}_{1}^{0}(M)$ and $X \in \mathscr{T}_{0}^{1}(M)$, we have 


$$
(\theta(X))^{A}=\theta^{A}\left(X^{A}\right)
$$

Proof. The function $\theta(X): M \rightarrow R$ can be written as $\theta(X)=\theta \circ X$, where $X: M \rightarrow M^{D}$ and $\theta: M^{D} \rightarrow R$. Hence we have $(\theta(X))^{A}=(\theta \circ X)^{A}=\theta^{A} \circ X^{A}$ $=\theta^{A}\left(X^{A}\right)$.

Lemma 4.6. For $\theta \in \mathscr{T}_{1}^{0}(M), a \in A$ and $\tilde{X} \in T_{x^{\prime}}\left(M^{A}\right)$, we have

$$
\theta^{A}(a \cdot \tilde{X})=a \cdot \theta^{A}(\tilde{X}) .
$$

Proof. Since $\theta(t \cdot X)=t \theta(X)$ for $t \in R$ and $X \in T(M)$ we have $(\theta \circ \mu)(t, X)$ $=\theta(t X)=\mu_{0}(t, \theta(X))=\mu_{0}(1 \times \theta)(t, X)$. Hence $(\theta \circ \mu)^{A}=\left(\mu_{0}\right)^{A} \circ\left(1 \times \theta^{A}\right)=$ $\mu_{A} \circ\left(1 \times \theta^{A}\right)$, which implies (4.5).

Since $\theta^{A}$ is an $A$-valued 1 -form on $M^{A}$, we can consider it as $\theta^{A} \in \mathscr{T}_{1}^{0}\left(M^{A}\right)$ $\otimes A$. We can easily verify

Lemma 4.7. $\mathscr{T}_{*}^{0}\left(M^{A}\right) \otimes A$ becomes an associative graded algebra over $A$ with the multiplication:

$$
\left(K_{1} \otimes a_{1}\right) \otimes\left(K_{2} \otimes a_{2}\right)=K_{1} \otimes K_{2} \otimes\left(a_{1} a_{2}\right)
$$

for $K_{1}, K_{2} \in \mathscr{T}_{*}^{0}\left(M^{A}\right)=\sum_{q} \mathscr{T}_{q}^{0}\left(M^{A}\right)$ and $a_{1}, a_{2} \in A$.

Lemma 4.8. The map $L: \mathscr{T}_{*}^{0}(M) \rightarrow \mathscr{T}_{*}^{0}\left(M^{A}\right) \otimes A$ defined by $L\left(\theta_{1} \otimes \ldots\right.$ $\left.\otimes \theta_{q}\right)=\theta_{1}{ }^{A} \otimes \cdots \otimes \theta_{q}{ }^{A}$ for $\theta_{i} \in \mathscr{T}_{1}^{0}(M)$ is an algebra homomorphism.

Proof. Let $L:\left(\mathscr{T}_{1}^{0}(M)\right)^{q} \rightarrow \mathscr{T}_{q}^{0}\left(M^{A}\right) \otimes A$ be defined by $L\left(\theta_{1}, \cdots, \theta_{q}\right)=\theta_{1}{ }^{A}$ $\otimes \cdots \otimes \theta_{q}{ }^{A}$. It is easily checked that $L\left(f_{1} \theta_{1}, \cdots, f_{q} \theta_{q}\right)=\left(f_{1} \cdots f_{q}\right)^{A} L\left(\theta_{1}, \cdots\right.$, $\left.\theta_{q}\right)$ for $f_{i} \in \mathscr{T}_{0}^{0}(M), \theta_{i} \in \mathscr{T}_{1}^{0}(M)$. Hence there exists a map $L: \mathscr{T}_{q}^{0}(M) \rightarrow \mathscr{T}_{q}^{0}\left(M^{A}\right)$ $\otimes A$ such that $L\left(\theta_{1} \otimes \cdots \otimes \theta_{q}\right)=L\left(\theta_{1}, \cdots, \theta_{q}\right)$. Now it is easy to see that $L$ is an algebra homomorphism.

\section{Lifting of $(1,1)$-tensor fields}

Let $K \in \mathscr{T}_{1}^{1}(M)$ be a $(1,1)$-tensor field on $M$. Then $K$ can be considered as a map $K: M^{D} \rightarrow M^{D}$ such that $\pi \circ K=\pi$. Then $K^{A}:\left(M^{D}\right)^{A} \rightarrow\left(M^{D}\right)^{A}$ can be considered as $K^{A}:\left(M^{A}\right)^{D} \rightarrow\left(M^{A}\right)^{D}$.

Lemma 5.1. $K^{A}$ is a $(1,1)$-tensor field on $M^{A}$.

Proof. Since the problem is local, we assume $K=\sum \theta_{i} \otimes Y^{i}$ with $\theta_{i} \in$ $\mathscr{T}_{1}^{0}(M)$ and $Y^{i} \in \mathscr{T}_{0}^{1}(M)$. Then

$$
\begin{aligned}
K(X)=\sum \theta_{i}(X) Y^{i} & =\sum \mu\left(\theta_{i}(X),\left(Y^{i} \circ \pi\right)(X)\right) \\
& =\left(\alpha_{r} \circ\left(\mu \circ\left(\theta_{1}, Y^{1} \circ \pi\right), \cdots, \mu \circ\left(\theta_{r}, Y^{r} \circ \pi\right)\right)\right)(X),
\end{aligned}
$$

where $\alpha_{r}: \boldsymbol{R}^{r} \rightarrow \boldsymbol{R}$ is the addition $\alpha_{r}\left(a_{1}, \cdots, a_{r}\right)=a_{1}+\cdots+a_{r}$ for $a_{i} \in \boldsymbol{R}$. Hence we have

$$
K^{A}=\left(\alpha_{r}\right)^{A} \circ\left(\mu^{A} \circ\left(\theta_{1}{ }^{A},\left(Y^{1}\right)^{A} \circ \pi^{A}\right), \cdots, \mu^{A} \circ\left(\theta_{r}{ }^{A},\left(Y^{r}\right)^{A} \circ \pi^{A}\right)\right),
$$

which implies 


$$
K^{A}(\tilde{X})=\sum \theta_{i}^{A}(\tilde{X}) \cdot\left(Y^{i}\right)^{A}
$$

for $\tilde{X} \in\left(M^{A}\right)^{D}$. Thus $K^{A} \in \mathscr{T}_{1}^{1}\left(M^{A}\right)$.

Lemma 5.2. For $K \in \mathscr{T}_{1}^{1}(M), X \in \mathscr{T}_{0}^{1}(M)$ and $a \in A$, we have

$$
K^{A}\left(a \cdot X^{A}\right)=a \cdot(K(X))^{A} .
$$

Proof. As before, we can assume $K=\sum \theta_{i} \otimes Y_{i}$. Then

$$
\begin{aligned}
K^{A}\left(a \cdot X^{A}\right) & =\sum \theta_{i}{ }^{A}\left(a X^{A}\right) \cdot\left(Y^{i}\right)^{A}=\sum a \cdot \theta_{i}{ }^{A}\left(X^{A}\right)\left(Y^{i}\right)^{A} \\
& =a \cdot \sum\left(\theta_{i}(X)\right)^{A}\left(Y^{i}\right)^{A}=a \cdot\left(\sum \theta_{i}(X) Y^{i}\right)^{A}=a \cdot(K(X))^{A} .
\end{aligned}
$$

Theorem 5.3. Let $J \in \mathscr{T}_{1}^{1}(M)$ be an almost complex structure on $M$. Then $J^{A}$ is an almost complex structure on $M^{A}$. Moreover, $J^{A}$ is integrable if and only if $\mathrm{J}$ is.

Proof. Let $I$ be the $(1,1)$-tensor field of identity maps of $T_{x} M$ for $x \in M$. Since $I=\sum d x_{i} \otimes \partial / \partial x_{i}$ locally, we get, for $\tilde{X} \in\left(M^{A}\right)^{D}$,

$$
\begin{aligned}
I^{A}(\tilde{X}) & =\sum\left(d x_{i}\right)^{A}(\tilde{X}) \otimes\left(\frac{\partial}{\partial x_{i}}\right)^{A}=\sum d x_{i}{ }^{A}(\tilde{X}) \cdot \frac{\partial}{\partial x_{i, 0}} \\
& =\sum d x_{i, \lambda}(\tilde{X}) B^{\lambda} \frac{\partial}{\partial x_{i, 0}}=\sum d x_{i, \lambda(\tilde{X})} \cdot \frac{\partial}{\partial x_{i, \lambda}}=\tilde{X}
\end{aligned}
$$

where we have used (3.9) and (4.5). Thus we have $J^{A} \circ J^{A}=(J \circ J)^{A}=(-I)^{A}$ $=-I^{A}=-\tilde{I}$, where $\tilde{I}$ is the $(1,1)$-tensor field of identity maps of $M^{A}$. Hence $J^{A}$ is an almost complex structure on $M^{A}$.

Next, $J$ is integrable if and only if

$$
J[X, Y]=[J X, Y]+[X, J Y]+J[J X, J Y]
$$

for every $X, Y \in \mathscr{T}_{0}^{1}(M)$. Using Lemmas 3.12 and 5.2 we have

$$
\begin{aligned}
J^{A}\left[a x^{A}, b Y^{A}\right] & =J^{A}\left(a b[X, Y]^{A}\right)=(a b)(J[X, Y])^{A} \\
& =(a b)\left\{\left[J^{A} X^{A}, Y^{A}\right]+\left[X^{A}, J^{A} Y^{A}\right]+J^{A}\left[J^{A} X^{A}, J^{A} Y^{A}\right]\right\} \\
& =\left[J^{A}\left(a X^{A}\right), b Y^{A}\right]+\left[a X^{A}, J^{A}\left(b Y^{A}\right)\right]+J^{A}\left[J^{A}\left(a X^{A}\right), J^{A}\left(b Y^{A}\right)\right]
\end{aligned}
$$

for $a, b \in A$. Since $T_{x^{\prime}} M^{A}$ is a free $A$-module (Corollary 3.10), we conclude that $J^{A}$ is integrable. Conversely, if $J^{A}$ is integrable, we get

$$
(J[X, Y])^{A}=([J X, Y]+[X, J Y]+J[J X, J Y])^{A}
$$

for $X, Y \in \mathscr{T}_{0}^{1}(M)$, which implies (5.1), and hence $J$ is integrable. 


\section{Prolongations of affine connections}

Let $\nabla$ be the covariant differentiation defined by an affine connection of $M$. In the sequel, for the sake of convenience of notation, we shall denote by $\nabla(X, K)$ the covariant differentiation of a tensor field $K$ on $M$ with respect to $X \in \mathscr{T}_{0}^{1}(M)$, i.e.,

$$
\nabla(X, K)=\nabla_{X} K
$$

Theorem 6.1. There exists one and only one affine connection on $M^{A}$ whose covariant differentiation $\tilde{\nabla}$ satisfies the following condition

$$
\tilde{\nabla}_{a X^{A}} b Y^{A}=(a b)\left(\nabla_{X} Y\right)^{A}
$$

for every $X, Y \in \mathscr{T}_{0}^{1}(M)$ and $a, b \in A$.

Proof. Take a coordinate neighborhood $U$ with coordinate system $\left\{x_{1}, \cdots\right.$, $\left.x_{n}\right\}$ and let $\Gamma_{i j}{ }^{k}$ be the connection components of $\nabla$ with respect to $\left\{x_{1}, \cdots, x_{n}\right\}$, i.e.,

$$
\nabla\left(\frac{\partial}{\partial x_{i}}, \frac{\partial}{\partial x_{j}}\right)=\sum \Gamma_{i}{ }_{j} \frac{\partial}{\partial x_{k}}
$$

for $i, j=1, \cdots, n$. Let $\Gamma_{i j}^{\prime}{ }^{k}$ be the connection components of $\nabla$ with respect to another coordinate system $\left\{y_{1}, \cdots, y_{n}\right\}$ on $U$. Then we have the following equalities :

$$
\Gamma_{i}^{\prime k}{ }_{j}=\sum \frac{\partial x_{b}}{\partial y_{i}} \frac{\partial x_{c}}{\partial y_{j}} \frac{\partial y_{k}}{\partial x_{a}} \Gamma_{b}{ }^{a}{ }_{c}+\sum \frac{\partial^{2} x_{a}}{\partial y_{i} \partial y_{j}} \frac{\partial y_{k}}{\partial x_{a}}
$$

for $i, j=1,2, \cdots, n$ (cf. for instance [3, p. 27]). Let $\left\{x_{i, \lambda} \mid i=1, \cdots, n ; \lambda=\right.$ $0,1, \cdots, N\}$ (resp. $\left\{y_{i, \lambda}\right\}$ ) be the induced coordinate system on $\pi_{A}{ }^{-1}(U)$. Define $\tilde{\Gamma}_{(i, \lambda)}{ }_{(k, \nu)}^{(j, \mu)}$ by

$$
\sum \tilde{\Gamma}_{(i, \lambda)}^{(k, \nu)}{ }_{(j, \mu)} B^{\nu}=B^{\lambda} B^{\mu}\left(\Gamma_{i j}{ }^{k}\right)^{A}
$$

for $i, j, k=1, \cdots, n ; \lambda, \mu, \nu=0,1, \cdots, N$, where $\left\{B^{0}=1, B^{1}, \cdots, B^{N}\right\}$ is a basis of $A$ as in $\S 1$.

We shall now prove that there exists a connection $\tilde{\nabla}$ whose connection components with respect to $\left\{x_{i, \lambda}\right\}$ are given by (6.4). To prove this we have to prove the following equalities (6.5) similar to (6.3):

$$
\begin{aligned}
\tilde{\Gamma}_{(i, \nu)}^{\prime(k, \lambda)}{ }_{(j, \mu)}^{(j)}= & \sum \frac{\partial x_{b, \beta}}{\partial y_{i, \nu}} \frac{\partial x_{c, r}}{\partial y_{j, \mu}} \frac{\partial y_{k, \lambda}}{\partial x_{a, \alpha}} \tilde{\Gamma}_{(b, \beta)}^{(a, \alpha)}{ }_{(c, r)} \\
& +\sum \frac{\partial^{2} x_{a, \alpha}}{\partial y_{i, \nu} \partial y_{j, \mu}} \frac{\partial y_{k, \lambda}}{\partial x_{a, \alpha}}
\end{aligned}
$$


for $i, j, k=1, \cdots, n ; \lambda, \mu, \nu=0,1, \cdots, N$, where $\tilde{\Gamma}_{(i, \nu)}^{\prime(k, \lambda)}\left({ }_{j, \mu)}\right.$ denote the connection components of $\tilde{V}$ with respect to the coordinate system $\left\{y_{i, \lambda}\right\}$. Denoting the right hand side of (6.5) by $\tilde{\Gamma}_{(i, \nu)}^{*(k, \lambda)}{ }_{(j, \mu)}$ and using Lemmas 3.8 and 3.9, we calculate as follows:

$$
\begin{aligned}
\sum \tilde{\Gamma}_{(i, \nu)}^{*}{ }_{(j, \mu)}^{(k, \lambda)} B^{\lambda} & \\
& =\frac{\partial x_{b, \beta}}{\partial y_{i, \nu}} \frac{\partial x_{c, r}}{\partial y_{j, \mu}}\left(\frac{\partial y_{k}}{\partial x_{a}}\right)^{A} B^{\alpha} \tilde{\Gamma}_{(b, \beta)}{ }^{(a, \alpha)}{ }_{(c, r)}+\frac{\partial^{2} x_{a, \alpha}}{\partial y_{i, \nu} \partial y_{j, \mu}}\left(\frac{\partial y_{k}}{\partial x_{a}}\right)^{A} B^{\alpha} \\
& =\frac{\partial x_{b, \beta}}{\partial y_{i, \nu}} \frac{\partial x_{c, r}}{\partial y_{j, \mu}}\left(\frac{\partial y_{k}}{\partial x_{a}}\right)^{A} B^{\beta} B^{\gamma}\left(\Gamma_{b}{ }^{a}{ }_{c}\right)^{A}+\frac{\partial}{\partial y_{i, \nu}}\left(\frac{\partial x_{a, \alpha}}{\partial y_{j, \mu}} B^{\alpha}\right)\left(\frac{\partial y_{k}}{\partial x_{a}}\right)^{A} \\
& =\left(\frac{\partial x_{b}}{\partial y_{i}}\right)^{A} B^{\nu}\left(\frac{\partial x_{c}}{\partial y_{j}}\right)^{A} B^{\mu}\left(\frac{\partial y_{k}}{\partial x_{a}}\right)^{A}\left(\Gamma_{b}{ }^{a}{ }_{c}\right)^{A}+\frac{\partial}{\partial y_{i, \nu}}\left(\frac{\partial x_{a}}{\partial y_{j}}\right)^{A} B^{\mu}\left(\frac{\partial y_{k}}{\partial x_{a}}\right)^{A} \\
& =\left(\frac{\partial x_{b}}{\partial y_{i}}\right)^{A} B^{\nu}\left(\frac{\partial x_{c}}{\partial y_{j}}\right)^{A} B^{\mu}\left(\frac{\partial y_{k}}{\partial x_{a}}\right)^{A}\left(\Gamma_{b}{ }^{a}{ }_{c}\right)^{A}+\left(\frac{\partial^{2} x_{a}}{\partial y_{i} \partial y_{j}}\right)^{A} B^{\nu} B^{\mu}\left(\frac{\partial y_{k}}{\partial x_{a}}\right)^{A} \\
& =B^{\nu} B^{\mu}\left(\Gamma_{i}^{\prime \prime}{ }_{j}\right)^{A}=\tilde{\Gamma}_{(i, \nu)}^{\prime}{ }_{(j, \mu)}^{(k, \lambda)} B^{\lambda},
\end{aligned}
$$

which implies (6.5).

Thus we have proved the existence of $\tilde{\nabla}$ whose connection components with respect to $\left\{x_{i, \lambda}\right\}$ are given by (6.4).

Next, we shall prove (6.1) for $X=\partial / \partial x_{i}, Y=\partial / \partial x_{j}$, and $a=B^{\lambda}, b=B^{\mu}$. We calculate as follows:

$$
\begin{gathered}
\tilde{\nabla}\left(B^{\lambda}\left(\frac{\partial}{\partial x_{i}}\right)^{A}, B^{\mu}\left(\frac{\partial}{\partial x_{j}}\right)^{A}\right)=\tilde{\nabla}\left(\frac{\partial}{\partial x_{i, \lambda}}, \frac{\partial}{\partial x_{j, \mu}}\right)=\tilde{\Gamma}_{(i, \lambda)}^{(k, \nu)}{ }_{(j, \mu)} \frac{\partial}{\partial x_{k, \nu}} \\
=\tilde{\Gamma}_{(i, \lambda)}{ }_{(k, \nu)}^{(k, \mu)} B^{\nu}\left(\frac{\partial}{\partial x_{k}}\right)^{A}=B^{\lambda} B^{\mu}\left(\Gamma_{i}{ }^{k}{ }_{j}\right)^{A}\left(\frac{\partial}{\partial x_{k}}\right)^{A} \\
=B^{\lambda} B^{\mu}\left(\Gamma_{i}{ }^{k}{ }_{j} \frac{\partial}{\partial x_{k}}\right)^{A}=B^{\lambda} B^{\mu}\left(\nabla\left(\frac{\partial}{\partial x_{i}}, \frac{\partial}{\partial x_{j}}\right)\right)^{A},
\end{gathered}
$$

which proves (6.1) for $X=\partial / \partial x_{i}, Y=\partial / \partial x_{j}$ and $a=B^{\lambda}, b=B^{\mu}$ and hence for arbitrary $a, b \in A$.

Now put $X_{i}=\partial / \partial x_{i}$ for $i=1, \cdots, n$. We shall prove (6.1) for $X=f X_{i}$, $Y=X_{j}$ with $f \in \mathscr{T}_{0}^{0}(U)$. We calculate as follows :

$$
\begin{aligned}
\tilde{\nabla}\left(a X^{A}, b Y^{A}\right) & =\tilde{\nabla}\left(a\left(f \cdot X_{i}\right)^{A}, b \cdot X_{j}{ }^{A}\right) \tilde{V}\left(a f^{A} \cdot X_{i}{ }^{A}, b \cdot X_{j}{ }^{A}\right) \\
& =a f^{A} \tilde{\nabla}\left(X_{i}{ }^{A}, b X_{j}{ }^{A}\right)=a b f^{A}\left(\nabla\left(X_{i}, X_{j}\right)\right)^{A} \\
& =a b\left(f \nabla\left(X_{i}, X_{j}\right)\right)^{A}=a b\left(\nabla\left(f X_{i}, X_{j}\right)\right)^{A}=a b(\nabla(X, Y))^{A},
\end{aligned}
$$

which proves our assertion. Therefore we see that (6.1) holds for $X \in \mathscr{T}_{0}^{1}(M)$ and $Y=X_{j}$ with $j=1, \cdots, n$. Next we prove that (6.1) holds for $X \in \mathscr{T}_{0}^{1}(M)$ and $Y=f \cdot X_{j}$ with $f \in \mathscr{T}_{0}^{0}(U)$ and $j=1, \cdots, n$. We calculate as follows : 


$$
\begin{aligned}
\tilde{\nabla}\left(a \cdot X^{A}, b \cdot Y^{A}\right) & =\tilde{\nabla}\left(a \cdot X^{A}, b\left(f \cdot X_{j}\right)^{A}\right)=\tilde{\nabla}\left(a X^{A}, b f^{A} \cdot X_{j}{ }^{A}\right) \\
& =f^{A} \nabla_{a X^{A}} b X_{j}{ }^{A}+a X^{A}\left(b f^{A}\right) X_{j}{ }^{A}=f^{A} a b\left(\nabla_{X} Y_{j}\right)^{A}+a b(X f)^{A} X_{j}{ }^{A} \\
& =a b\left(f \nabla_{X} Y_{j}+X f \cdot X_{j}\right)^{A}=a b\left(\nabla_{X} f Y_{j}\right)^{A}=a b(\nabla(X, Y))^{A}
\end{aligned}
$$

where we have used Lemmas 3.5 and 3.8.

Thus we have proved (6.1) for any $X, Y \in \mathscr{T}_{0}^{1}(M)$ and $a, b \in A$. The uniqueness of such $\tilde{\nabla}$ follows from Lemma 3.9 .

Definition 6.2. The unique affine connection $\tilde{\nabla}$ in Theorem 6.1 will be called the prolongation of $\nabla$ to $M^{A}$ and will be denoted by $\tilde{\nabla}=\nabla^{A}$.

Theorem 6.3. Let $T$ and $R$ (resp. $\tilde{T}$ and $\tilde{R}$ ) be the torsion and curvature tensor fields of $\nabla$ (resp. $\left.\tilde{\nabla}=\nabla^{A}\right)$. Then according as $T=0, \nabla T=0, R=0$ or $\nabla R=0$, we have $\tilde{T}=0, \tilde{\nabla} \tilde{T}=0, \tilde{R}=0$ or $\tilde{\nabla} \tilde{R}=0$ and vice versa. In particular, if $M$ is locally affine symmetric with respect to $\nabla$, so is $M^{A}$ with respect to $\tilde{\nabla}=\nabla^{A}$.

Proof. First we prove

$$
\tilde{T}\left(a X^{A}, b Y^{A}\right)=a b(T(X, Y))^{A}
$$

for $X, Y \in \mathscr{T}_{0}^{1}(M)$ and $a, b \in A$.

In fact, by the definition of $\tilde{T}$, Lemma 3.12 and (6.1) we get

$$
\begin{aligned}
\tilde{T}\left(a X^{A}, b Y^{A}\right) & =\tilde{\nabla}_{a X^{A}} b Y^{A}-\tilde{\nabla}_{b Y^{A}} a X^{A}-\left[a X^{A}, b Y^{A}\right] \\
& =(a b)\left(\nabla_{X} Y-\nabla_{Y} X-[X, Y]\right)^{A}=a b(T(X, Y))^{A}
\end{aligned}
$$

Thus we see that $T=0$ if and only if $\tilde{T}=0$ (cf. Corollary 3.10).

Similarly we know that $\tilde{R}\left(a X^{A}, b Y^{A}, c Z^{A}\right)=(a b c)(R(X, Y, Z))^{A}$ for $X, Y, Z$ $\in \mathscr{T}_{0}^{1}(M)$ and $a, b, c \in A$, from which we see that $R=0$ if and only if $\tilde{R}=0$. The proof for the case $\nabla T$ and $\nabla R$ is similar.

\section{Affine symmetric spaces}

Lemma 7.1. Let $\Phi$ be a diffeomorphism of $M$ onto $M^{\prime}$, and let $X \in \mathscr{T}_{0}^{1}(M)$ and $a \in A$. Then we have

$$
\left(T \Phi^{A}\right)\left(a X^{A}\right)=a((T \Phi) X)^{A} .
$$

Proof. Take $f \in C^{\infty}(M)$. We have

$$
\begin{aligned}
\left(\Phi^{A}\right)^{D}\left(a X^{A}\right) f^{A} & =\left(a X^{A}\right)\left(f^{A} \circ \Phi^{A}\right)=\left(a X^{A}\right)(f \circ \Phi)^{A}=a \cdot X^{A}(f \circ \Phi)^{A} \\
& =a \cdot(X(f \circ \Phi))^{A}=a \cdot\left(\left(\Phi^{D} X\right) f\right)^{A}=a \cdot\left(\left(\Phi^{D} X\right)^{A} f^{A}\right) \\
& =\left(a\left(\Phi^{D} X\right)^{A}\right) f^{A}
\end{aligned}
$$

from which follows (7.1).

Lemma 7.2. Let $\nabla$ (resp. $\nabla^{\prime}$ ) be an affine connection on $M$ (resp. on $M^{\prime}$ ) 
and let $\Phi$ be a diffeomorphism of $M$ onto $M^{\prime}$ transforming $\nabla$ onto $\nabla^{\prime}$. Then $\Phi^{A}$ transforms $\nabla^{A}$ onto $\nabla^{\prime A}$.

Proof. Take $X, Y \in \mathscr{T}_{0}^{1}(M)$. Then we have, for $a, b \in A$,

$$
\begin{aligned}
T \Phi^{A}\left(\nabla_{a X^{A}}^{A} b Y^{A}\right) & =T \Phi^{A}\left(a b \nabla_{X} Y\right)^{A}=a b\left(T \Phi\left(\nabla_{X} Y\right)\right)^{A}=a b\left(\nabla_{T \Phi X}^{\prime} T \Phi Y\right)^{A} \\
& =\nabla_{a(T \Phi X)^{A}}^{A} b(T \Phi Y)^{A}=\nabla_{T \Phi A\left(a X^{A}\right)}^{\prime} T \Phi^{A}\left(b Y^{A}\right),
\end{aligned}
$$

where we have used Lemma 7.1. Since $X, Y, a, b$ are arbitrary, Lemma 7.2 follows.

Lemma 7.3. Let $X \in \mathscr{T}_{0}^{1}(M)$, and $x_{0} \in M$. Assume $X_{x_{0}}=0$. Then $\left(X^{A}\right)_{\tilde{x}_{0}}$ $=0$, where $\tilde{x}_{0} \in M^{A}$ is defined by $\tilde{x}_{0}(f)=f\left(x_{0}\right)$ for $f \in C^{\infty}(M)$.

Proof. Let $\Phi^{t}$ be a local one-parameter group of local diffeomorphisms around $x_{0}$ generated by $X$. Then $X^{A}$ generates the local group $\left(\Phi^{t}\right)^{A}$ around $\tilde{x}_{0}$ (cf. Remark 3.13). Since $\Phi^{t}\left(x_{0}\right)=x_{0}$, we get $\left(\Phi^{t}\right)^{A}\left(\tilde{x}_{0}\right)=\tilde{x}_{0}$ and therefore $\left(X^{A}\right)_{\tilde{x}_{0}}=0$.

Lemma 7.4. Let $\Phi: M \rightarrow M$ be a diffeomorphism such that there exist $x_{0} \in M$ and $\alpha \in R$ with $\Phi\left(x_{0}\right)=x_{0}$ and $T_{x_{0}} \Phi=\alpha \cdot 1_{T_{x_{0} M}}$. Then $T_{\tilde{x}_{0}} \Phi^{A}=$ $\alpha \cdot 1_{T_{\tilde{x}_{0} M^{A}} \text {. }}$

Proof. Let $\left\{x_{1}, \cdots, x_{n}\right\}$ be a local coordinate system around $x_{0}$. By Lemma 7.1 we have $T \Phi^{A}\left(\partial / \partial x_{i}\right)^{A}=\left(T \Phi\left(\partial / \partial x_{i}\right)\right)^{A}$ for $i=1, \cdots, n$. Hence we get

$$
T \Phi^{A}\left(\left(\partial / \partial x_{i}\right)_{\tilde{x}_{0}}^{A}\right)=\left(T \Phi^{A}\left(\partial / \partial x_{i}\right)^{A}\right)_{\tilde{x}_{0}}=\left(T \Phi\left(\partial / \partial x_{i}\right)\right)_{\tilde{x}_{0}}^{A} .
$$

Put $X=T \Phi\left(\partial / \partial x_{i}\right)-\alpha\left(\partial / \partial x_{i}\right)$. Then $X$ is a vector field around $x_{0}$ on $M$ with $X_{x_{0}}=0$. Therefore by Lemma 7.3 we get $\left(X^{A}\right)_{\tilde{x}_{0}}=0$, which implies

$$
\left(T \Phi\left(\partial / \partial x_{i}\right)\right)_{\tilde{x}_{0}}^{A}=\left(\alpha\left(\partial / \partial x_{i}\right)\right)_{\tilde{x}_{0}}^{A}=\alpha \cdot\left(\partial / \partial x_{i}\right)_{\tilde{x}_{0}}^{A} .
$$

Take an arbitrary $a \in A$. Then we have

$$
T \Phi^{A}\left(a\left(\partial / \partial x_{i}\right)\right)_{\tilde{x}_{0}}^{A}=a \cdot\left(T \Phi\left(\partial / \partial x_{i}\right)\right)_{\tilde{x}_{0}}^{A}=a \cdot \alpha\left(\partial / \partial x_{i}\right)_{\tilde{x}_{0}}^{A}=\alpha \cdot\left(a\left(\partial / \partial x_{i}\right)_{\tilde{x}_{0}}^{A}\right) .
$$

Since $\left\{a\left(\partial / \partial x_{i}\right)^{A} \mid a \in A\right\}$ span the tangent space $T_{\tilde{x}_{0}} M^{A}$ (cf. Lemma 3.9), we get $T_{\tilde{x}_{0}} \Phi^{A}=\alpha \cdot 1_{T_{\tilde{x}_{0} M}{ }^{A}}$.

Corollary 7.5. Let $\Phi$ be the affine symmetry at a point $x_{0} \in M$ with respect to an affine connection $\nabla$ on $M$. Then $\Phi^{A}$ is the affine symmetry of $M^{A}$ at $\tilde{x}_{0}$ with respect to $\nabla^{A}$.

Proof. Since $\Phi$ leaves $\nabla$ invariant, $\Phi^{A}$ leaves $\nabla^{A}$ invariant by Lemma 7.2. Next, since $\Phi$ is the affine symmetry we see that $T_{x_{0}} \Phi=-1_{T_{x_{0} M} M}$. Thus by Lemma 7.4 we get $T_{\tilde{x}_{0}} \Phi^{A}=-1_{T_{\tilde{x}_{0} M^{A}}}$, which means that $\Phi^{A}$ is the affine symmetry at $\tilde{x}_{0}$.

Proposition 7.6. Let $\nabla$ be an affine connection on $M$ and let $X \in T_{0}^{1}(M)$ be an infinitesimal affine transformation of $\nabla$. Then, for any $a \in A, a X^{A}$ is also an infinitesimal affine transformation of $\nabla^{A}$. 
Proof. A necessary and sufficient condition for $X$ to be an infinitesimal affine transformation of $M$ is that

$$
L_{X} \circ \nabla_{Y}-\nabla_{Y} \circ L_{X}=\nabla_{[X, Y]}
$$

for every $Y \in \mathscr{T}_{0}^{1}(M)$, where $L_{X}$ (or $L(X)$ ) denotes the Lie derivation with respect to $X$. Therefore we have to prove

$$
L\left(a \cdot X^{A}\right)\left(\nabla^{A}(\tilde{Y}, K)\right)-\nabla^{A}\left(\tilde{Y}, L\left(a X^{A}\right) K\right)=\nabla^{A}\left(\left[a X^{A}, \tilde{Y}\right], K\right)
$$

for every $K \in \mathscr{T}\left(M^{A}\right)$ and $\tilde{Y} \in \mathscr{T}_{0}^{1}\left(M^{A}\right)$. To prove (7.2) it suffices to prove (7.2) for the special cases, where $\tilde{Y}=b Y^{A}$ with $Y \in \mathscr{T}_{0}^{1}(M), b \in A$, and $K=$ $c \cdot Z^{A}$ or $\theta^{A}$ with $Z \in \mathscr{T}_{0}^{1}(M), \theta \in \mathscr{T}_{1}^{0}(M)$ and $c \in A$. Moreover, to prove (7.2) for $K=\theta^{A}$, it suffices to prove it for $\theta=d f$ with $f \in \mathscr{T}_{0}^{0}(M)$.

If $K=c Z^{A}$, we calculate as follows :

$$
\begin{aligned}
L_{a X^{A}} \tilde{\nabla}_{b Y^{A}} c Z^{A}-\tilde{\nabla}_{b Y^{A}} L_{a X^{A}} c Z^{A} & =\left[a X^{A}, b c\left(\nabla_{Y} Z\right)^{A}\right]-\tilde{\nabla}_{b Y^{A}}\left[a X^{A}, c Z^{A}\right] \\
& =a b c\left[X, \nabla_{Y} Z\right]^{A}-b a c\left(\nabla_{Y}[X, Z]\right)^{A} \\
& =a b c\left(\left(L_{X} \nabla_{Y}-\nabla_{Y} L_{X}\right) Z\right)^{A}=a b c\left(\nabla_{[X, Y]} Z\right)^{A} \\
& =\tilde{\nabla}_{\left[a X^{A}, b Y^{A}\right]} c Z^{A} .
\end{aligned}
$$

If $K=d f^{A}$, we have

$$
\begin{aligned}
L_{a X^{A}} & \tilde{\nabla}_{b Y^{A}}\left(d f^{A}\right)-\tilde{\nabla}_{b Y^{A}} L_{a X^{A}}\left(d f^{A}\right)\left(c Z^{A}\right) \\
= & \left(a X^{A}\right)\left(\tilde{\nabla}_{b Y^{A}} d f^{A}\right)\left(c Z^{A}\right)-\left(\tilde{\nabla}_{b Y^{A}} d f^{A}\right)\left(a X^{A}, c Z^{A}\right)-\left(\tilde{\nabla}_{b Y^{A}} d\left(a X^{A} f^{A}\right)\right)\left(c Z^{A}\right) \\
= & \left(a X^{A}\right)\left\{\left(b Y^{A}\right)\left(c Z^{A}\right)\left(f^{A}\right)-\left(\tilde{\nabla}_{b Y^{A}} c Z^{A}\right) f^{A}\right\} \\
& -\left\{b Y^{A}\left[a X^{A}, c Z^{A}\right] f^{A}-\left(\tilde{\nabla}_{b Y^{A}}\left[a X^{A}, c Z^{A}\right]\right) f^{A}\right\} \\
& -\left\{b Y^{A}\left(c Z^{A}\right)\left(a X^{A}\right) f^{A}-\left(\tilde{\nabla}_{b Y^{A}} c Z^{A}\right)\left(a X^{A}\right) f^{A}\right\} \\
= & a b c\left(\left\{L_{X} \circ \nabla_{Y}-\nabla_{Y^{\circ}} \circ L_{X}\right\}(d f)(Z)\right)^{A}=a b c\left(\left(\nabla_{[X, Y]}(d f)\right) Z\right)^{A} \\
= & a b c\left([X, Y] Z f-\left(\nabla_{[X, Y]} Z\right) f\right)^{A} \\
= & {\left[a X^{A}, b Y^{A}\right]\left(c Z^{A}\right) f^{A}-\left(\tilde{\nabla}_{\left[a X^{A}, b Y^{A}\right]} c Z^{A}\right) f^{A} } \\
= & \left(\tilde{\nabla}_{\left[a X^{A}, b Y^{A]}\right]}(d f)^{A}\right)\left(c Z^{A}\right) .
\end{aligned}
$$

Theorem 7.7. Let $M$ be an affine symmetric space with connection $\nabla$. Then $M^{A}$ is also an affine symmetric space with connection $\nabla^{A}$.

Proof. Let $G$ be the connected component of the group of all affine transformations of $M$. Then $G$ operates transitively on $M$. Let $X_{1}, \cdots, X_{m}$ be a basis of the Lie algebra $g$ of $G$. We denote by $X^{*} \in \mathscr{T}_{0}^{1}(M)$ the vector field induced by the one-parameter group of affine transformations generated by $X \in g$. Now we can show that $a X^{A}$ is a left invariant vector field on the Lie group $G^{A}$ and that $\left(a \cdot X^{A}\right)^{*}=a \cdot\left(X^{*}\right)^{A}$ holds for $a \in A$ (the detail will be omitted), which implies that $a \cdot\left(X^{*}\right)^{A}$ is complete, i.e., generates a global oneparameter group of affine transformations of $M^{A}$ (cf. Proposition 7.6). Hence 
we see that any element of $G^{A}$ is an affine transformation of $M^{A}$. The transitivity of $G$ shows that $\operatorname{dim}\left(\left\{X_{x}^{*} \mid X \in g\right\}\right)=\operatorname{dim} M$ for any $x \in M$, which implies

$$
\operatorname{dim}\left(\left\{\left(a \cdot\left(X^{*}\right)^{A}\right)_{x^{\prime}} \mid a \in A, X \in g\right\}\right)=\operatorname{dim} M^{A}
$$

for any $x^{\prime} \in M^{A}$ and hence the transitivity of $G^{A}$ on $M^{A}$ follows. On the other hand, by Corollary 7.5 we have an affine symmetry at $\tilde{x}_{0}$ of $M^{A}$ for $x_{0} \in M$. Hence $M^{A}$ is affinely symmetric.

Proposition 7.8. Let $\nabla$ be an affine connection on $M$. If $M^{A}$ is affinely symmetric with respect to $\nabla^{A}$, then $M$ is also so with respect to $\nabla$.

Proof. Consider the map $\zeta: M \rightarrow M^{A}$ defined by $(\zeta(x)) f=f(x)$ for $x \in M$, $f \in C^{\infty}(M)$. Let $\gamma: I \rightarrow M$ be a curve on $M$, where $I$ is an open interval in $R$. Put $\tilde{\gamma}=\zeta \circ \gamma$. From (6.4), we see that

$$
\tilde{\Gamma}_{(j, 0)}{ }^{(i, \lambda)}{ }_{(k, 0)}(\zeta(x))=\delta_{0}^{\lambda} \Gamma_{j k}^{i}(x)
$$

for $i, j, k=1, \cdots, n ; \lambda=0,1, \cdots, N$, from which we can verify that $\gamma$ is a geodesic on $M$ if and only if $\tilde{\gamma}$ is so on $M^{A}$. Further, we can conclude that the submanifold $\tilde{M}=\zeta(M)$ is a totally geodesic submanifold of $M^{A}$ with respect to $\nabla^{A}$ and that the induced affine connection $\nabla^{\prime}$ on $M$ is isomorphic with $\nabla$ by the diffeomorphism $\zeta: M \rightarrow \tilde{M}$.

Now, take an arbitrary point $x \in M$ and consider $\tilde{x}=\zeta(x) \in M^{A}$. Since $M^{A}$ is affinely symmetric, there exists an affine symmetry $\Phi$ of $M^{A}$ at $\tilde{x}$. Since

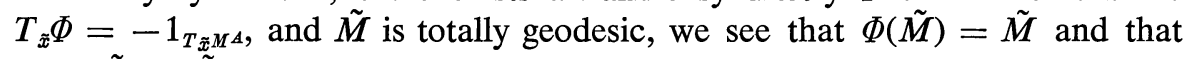
$\left.\Phi\right|_{\tilde{M}}: \tilde{M} \rightarrow \tilde{M}$ is an affine transformation of $\nabla^{\prime}$. Then $\left.\Phi\right|_{\tilde{M}}$ induces the affine symmetry $\Psi: M \rightarrow M$ of $M$ at $x$.

\section{References}

[1] C. Ehresmann, Les prolongements d'une variété différentiable. I, Calcul des jets, prolongement principal, C. R. Acad. Sci. Paris 233 (1951) 598-600.

[2] - Les prolongements d'une variété différentiable. II, L'espace des jets d'ordre $r$ de $\mathrm{V}_{\mathrm{n}}$ dans $\mathrm{V}_{\mathrm{m}}$, C. R. Acad. Sci. Paris 233 (1951) 777-779.

[ 3 ] S. Helgason, Differential geometry and symmetric spaces, Academic Press, New York, 1962.

[4] A. J. Ledger \& K. Yano, The tangent bundle of a locally symmetric space, J. London Math. Soc. 40 (1965) 487-492.

[5] A. Morimoto, Liftings of some types of tensor fields and connections to tangent bundles of $\mathrm{p}^{\mathrm{r}}$-velocities, Nagoya Math. J. 40 (1970) 13-31.

[6] - Prolongations of geometric structures, Lecture Notes, Nagoya University, 1969.

[ 7 ] A. Weil,'Théorie des points proches sur les variété différentiables, Colloq. Topologie Géométrie Différentielle, Strasbourg, 1953, 111-117.

[8] K. Yano \& S. Ishihara, Differential geometry of tangent bundles of order 2 , Kōdai Math. Sem. Rep. 20 (1968) 318-354.

[9] _- Tangent and cotangent bundles, Marcel Dekker, New York, 1973.

[10] K. Yano \& S. Kobayashi, Prolongations of tensor fields and connections to tangent bundles. I, J. Math. Soc. Japan 18 (1966) 194-210.

NAgOYa UNIVERSITY 